The annual incidence of leukaemias in children is 4.5 cases per 100,000 children. Acute lymphoblastic leukaemia accounts for approximately $77 \%$ of cases. The common presentations in this age group are pallor, irritability and bone pain. Leukaemia cutis can be a rare presenting sign of ALL (3\% of cases).

\section{P568 GITELMAN SYNDROME: A RARE CAUSE OF HYPOKALEMIA}

Jan Roux, Amani Abdalla*, Muhammad Pervaiz. University Hospital Kerry, Tralee, Ireland

10.1136/archdischild-2019-epa.902

Gitelman syndrome is a rare autosomal recessive renal tubular disorder characterized by hypoglycemia, metabolic alkalosis, hypomagnesemia and hypocalciurea, most often it is caused by a mutation in the solute carrier family 21 member 3 (SLC12A3) gene. Prevalence is approximately 1:40000 and the prevalence of hetero zygotes is approximately $1 \%$ in Caucasian populations, making it one of the most inherited renal tubular disorders.

We report a case of 7 year old boy who presented to emergency department with 4 days history of abdominal pain.

Past history revealed that he always likes salty and pickled food, having intermittent weakness and low energy but no paraesthesia or muscle cramps.

He was born at term with uneventful neonatal course. His development is normal and is fully vaccinated.

There is no family history of renal disease, Dad has IDDM, and he has one healthy brother.

On examination he was a slim boy, weight was $21.2 \mathrm{~kg}$, height $119 \mathrm{~cm}$ (both between 9-25th centile) his vitals including blood pressure were normal. Systemic examination was normal apart from mild peri-umbilical tenderness. Abdominal X-ray showed faecal loading of colon and us abdomen was normal. He was referred for paediatric assessment by surgical team because of incidental finding of low serum potassium. His venous blood gas showed metabolic alkalosis and serum magnesium was low normal. ECG showed sinus rhythm.

He was admitted and started on intravenous fluids with added potassium chloride as well as oral $\mathrm{K}$ supplements.

Other investigations including early morning urine, albumin lcreatinine ratio, urine for electrolytes, urine amino acids and urine for retinol binding proteins (RBP) were all normal. Blood sample for genetics was sent for Gitelman syndrome.

His oral potassium dose was increased gradually while weaning on potassium supplement in intravenous fluids. $\mathrm{He}$ was discharged home on oral potassium after his serum potassium level improved and booked for repeat bloods in day ward. His repeat blood showed low serum magnesium, so he started on oral magnesium supplement. He also needed further increase in his potassium dose to maintain his serum level $\geq 2.8 \mathrm{mmol} / \mathrm{L}$. His genetics tests confirmed two mutations in the SLC12A3 gene consistent with autosomal recessive Gitelman Syndrome.

Currently he is doing well and under regular follow up with general paediatric and paediatric nephrology.

Although it is a rare disorder, Gitelman Syndrome should be considered in children with unexplained hypokalaemia, with adequate treatment patients usually have excellent prognosis.

\section{P569} DEJERINE-SOTTAS SYNDROME AND CRANIO-FACIAL DYSMORPHISMS: A CASE REPORT

${ }^{1}$ Alessandra Biagini*, 'Elena Nechita, ${ }^{2}$ Willie Reardon, 'Hilary Greaney, 'Ghia Harrison.

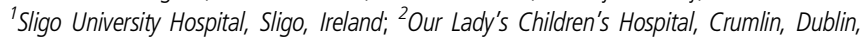
Ireland

10.1136/archdischild-2019-epa.903

Introduction Dejerine-Sottas syndrome (DSS) is a rare hereditary motor-sensor neuropathy transmitted as either autosomal dominant or recessive and classified as a severe degenerative neuropathy of the Charcot-Marie-Tooth type.

DSS is characterized by demyelination and remyelination features with an extensive nerve and root hypertrophy that results in a decreased nerve conduction velocity $(<10-12 \mathrm{~m} /$ s).

The hallmark clinical manifestations develop in early infancy with hypotonia, developmental motor delay and areflexia. Although arthrogryposis and spine deformities are frequent features, there are no direct associations with other dysmorphic features.

Case This report describes a rare association between DSS and cranio facial syndrome.

A 5 months old boy first presented in our Clinic with an early onset of motor symptoms manifested by congenital hypotonia, joint laxity particularly involving his lower limbs, failure to thrive, short stature and a significant psychomotor developmental delay.

On clinical examination he showed clear dysmorphic features with epicantic folds, hypertelorism, long philtrum, low set ears, downstanding of the eyes particularly the left eye and a convergent left eye squint.

Comment The presence of 'soft' clinical signs can distract from typical features of an underlying neurological syndrome leading to subsequent delayed or misdiagnosis in children with DSS.

In our experience, it is therefore important that Paediatricians can be aware of this possible association with this diagnosis and seek expert specialist geneticist advice if suspicious while simultaneously developing a management plan that supports and encourages attainment of maximal developmental process for the child.

\section{P570 AN UNUSUAL CAUSE OF RECURRENT VOMITING IN A SCHOOLBOY: DIETL'S CRISIS}

Ronan Callanan*, Alwyn Charles, Shozab Taj, Eoin Fitzgerald, Anne-Marie Murphy. UHL, Limerick, Ireland

\subsection{6/archdischild-2019-epa.904}

Backdround Congenital Pelvic Ureteric Junction Obstruction (PUJO) is caused by an intrinsic stenosis of the PUJ or external compression by an accessory renal artery.

Dietl's crisis is defined as episodic crampy upper abdominal pain, nausea and vomiting secondary to PUJO. Symptoms can be severe, increasing in nature with diuresis. The patient may also be asymptomatic between events.

Childhood vomiting is a regular presentation to Paediatric Emergency Departments (PED) worldwide

Aims Our aim is to report a case of an eight year old boy who presented to our PED with recurrent episodes of vomiting and abdominal pain, the ultimate diagnosis for which was Dietl's crisis. 
Methods We describe the clinical presentation, results of laboratory and radiological investigations, treatment and outcome to date.

A review of current available literature on this topic was also undertaken.

Results An 8 year old boy presented to the PED with severe nausea and vomiting with a cyclical pressure type right upper quadrant pain for 7 hours. On presentation the pain had reduced significantly. No symptoms of infection, no concerning vomit contents, diarrhoea or constipation.

This was the 10th similar episode in the previous 2 months. Previous investigations including blood panel, urine were normal and symptoms had resolved on attendance.

No abnormality was found on clinical exam.

Abdominal ultrasound demonstrated a large right sided hydronephrosis secondary to PUJO confirmed by CT KUB. A renogram demonstrated a partial obstruction and surgical management was planned electively.

Conclusion Our patient had experienced multiple episodes of Dietl's Crisis which had resolved independently. PUJO is not a common first time presentation in children of this age. We suggest that Paediatricians consider this diagnosis when the other more common differentials have been outruled while being mindful that clinical examinations, radiological and labaroratory investigations may be normal in between episodes of Dietl's crises.

\section{P571 FOETAL FEMUR FRACTURE AND EXTERNAL CEPHALIC VERSION: A CASE REPORT OF METAPHYSEAL INJURY AND LITERATURE REVIEW}

Dragos Valceanu*, Gerry Mackin, Patrick Mcllwaine, Isabela Govor. South West Acute Hospital, Enniskillen, UK

\subsection{6/archdischild-2019-epa.905}

Introduction External Cephalic Version (ECV) is a procedure used to turn a foetus from a breech or transverse position into a cephalic position prior to the onset of labour. It is recommended by the UK national guidelines to enable vaginal delivery for breech presentation of a singleton pregnancy. ECV is considered a safe manoeuvre when dealing with breech presentation and fractured femur is a rare but recognised complication of this procedure, with only 3 cases reported in the speciality literature.

Case We herein report a 4 months old boy, born at $38+4 / 40$ weeks gestational age by Kiwi extraction and episiotomy, with a birth weight of $4180 \mathrm{~g}$, Apgar score 8 at 1 minute and 9 at 5 minutes of life, no active resuscitation was required. The pregnancy was carefully monitored due to the maternal nutritional controlled gestational diabetes and foetopelvic unstable lie. Was breech at $37+5$ weeks and ECV manoeuvre performed, discharged home in stable condition afterwards. Postnatally paediatric team or parents did not raise any concern regarding pain or swelling of the knee. He was admitted to our Children's Ward at 10 days of age by a community midwife with suspected non-accidental injury. Physical examination revealed right lower limb in a flexed, antalgic position, non-ecchymotic swelling of the right knee, with tenderness to touch. Full examination revealed no additional injuries. Retrospective review of pictures taken by parents in the first day of life revealed a swollen knee being held in a flexed position.
Imaging investigations X-Ray Right knee reported florid callus formation surrounding the distal femoral metaphysis, representing an ossifying subperiosteal haematoma along with a bony fragment in relation to the anterolateral aspect of the distal metaphysis in keeping with an avulsion fracture.

Conclusion Based on antenatal, perinatal and postnatal history, revision of maternal case notes, and photographic evidence while being inpatient in the maternity ward, along with the presence of callus formation on X-Ray implied an injury older than 10 days. This suggests that the femoral fracture is most likely due to External Cephalic Version performed 6 days prior to delivery. A decision was made by the paediatric consultant to withhold any further safeguarding investigations as an aetiology for the child's fracture was detected.

\section{P572 PARTIAL DOUBLE TRISOMY 9 AND 13-FIRST REPORTED CASE IN MEDICAL LITERATURE}

${ }^{1}$ Qasim Mahmood, ${ }^{2,3}$ Birendra Rai ${ }^{*},{ }^{4}$ David Betts, ${ }^{1}$ Rizwan Khan. ${ }^{1}$ University Hospital Kerry, Kerry, Ireland; ${ }^{2}$ Mullingar Regional Hospital, Mullingar, Ireland; ${ }^{3}$ Northampton General Hospital, Northampton, UK; ${ }^{4}$ Our Lady's Children's Hospital, Crumlin, Ireland

\subsection{6/archdischild-2019-epa.906}

Background Both trisomy $9 p$ and partial trisomy $13 q$ have been recognised in past with characteristics clinical anomaly, our case is the first reported case of combined partial double trisomy involving chromosome $9 \mathrm{p}$ and $13 \mathrm{q}$. Phenotypic Characteristics vary based on the regions of the chromosome involved and the gene dosages effect. Characteristics of our index case would not only help clinician in genotype-phenotype correlation of any such future cases but would also add up to the already described consequences in offspring of balanced reciprocal translocations in either parents

Case report A female infant was born at 41 weeks gestation by normal delivery with birth weight $3.3 \mathrm{kgs}$. The pregnancy was uneventful. Baby had an episode of hypoglycaemia during very first day of life.

Physical examination of the baby revealed profound central hypotonia, head lag, low set ears, depressed nasal bridge and increased nuchal pad of fat. Cardiac examination revealed soft systolic murmur of grade $2 / 6$ which subsequently on echocardiography was noted to arise from a small atrial septal defect. Remainder systemic examination was within normal limits. Further course in the special care baby unit was complicated by recurrent apneas, desaturations and poor feeding. She also developed symptoms of cow milk protein's intolerance and gastro oesophageal reflux later on in life. Cranial ultrasound, Electroencephalography, MRI of the brain, renal ultrasound, sleep study, Laryngo bronchoscopy, chest and thoracic inlet Xrays were all normal. Array comparative genomic hybridisation, using a $60 \mathrm{~K}$ Agilent chip showed a gain of chromosome 9 material of approximately $30.9 \mathrm{Mb}$ at bands $9 \mathrm{p} 24.3-9 \mathrm{p} 21.1$ between base pair coordinates 204193 and 31104204, and an another gain of chromosome 13 material of approximately $11.2 \mathrm{Mb}$ at bands $13 \mathrm{q} 12.11-13 \mathrm{q} 12.3$ between base pair coordinates 20407295 and 31578124 , with the former representing the most proximal probe on this platform. Subsequent analysis of $G$ banded metaphase chromosomes demonstrated an abnormal female karyotype with an additional chromosome consistent with a der(13)t $(9 ; 13)(\mathrm{p} 21.1 ; \mathrm{q} 12)$.

Karyotypic analysis of the parents showed that the mother carried a balanced $t(9 ; 13)$ translocations. Therefore the transferred genetic defect in the index case was a product of $3: 1$ 